\title{
Identification and Characterization of Differentially Expressed Transcripts in the Gills of Freshwater Prawn (Macrobrachium rosenbergii) under Salt Stress
}

\author{
Hirak Kumar Barman, ${ }^{1}$ Swagat Kumar Patra, ${ }^{1}$ Varsha Das, ${ }^{1}$ Shibani Dutta Mohapatra, \\ Pallipuram Jayasankar, ${ }^{1}$ Chinmayee Mohapatra, ${ }^{1}$ \\ Ramya Mohanta, ${ }^{1}$ Rudra Prasanna Panda, ${ }^{1}$ and Surya Narayan Rath ${ }^{2}$ \\ ${ }^{1}$ Fish Genetics and Biotechnology Division, Central Institute of Freshwater Aquaculture (Indian Council of Agricultural Research), \\ Kausalyaganga, Bhubaneswar, Odisha 751002, India \\ ${ }^{2}$ Department of Bioinformatics, Centre for Post Graduate Studies, Orissa University of Agriculture \& Technology, Bhubaneswar, \\ Odisha 751003, India
}

Correspondence should be addressed to Hirak Kumar Barman, hkbarman68@hotmail.com

Received 27 October 2011; Accepted 15 November 2011

Academic Editor: Frédéric Becq

Copyright () 2012 Hirak Kumar Barman et al. This is an open access article distributed under the Creative Commons Attribution License, which permits unrestricted use, distribution, and reproduction in any medium, provided the original work is properly cited.

\begin{abstract}
The giant freshwater prawn, Macrobrachium rosenbergii, is an economically important species. It is a euryhaline shrimp, surviving in wide-range salinity conditions. A change in gene expression has been suggested as an important component for stress management. To better understand the osmoregulatory mechanisms mediated by the gill, a subtractive and suppressive hybridization (SSH) tool was used to identify expressed transcripts linked to adaptations in saline water. A total of 117 transcripts represented potentially expressed under salinity conditions. BLAST analysis identified $22 \%$ as known genes, $9 \%$ as uncharacterized showing homologous to unannotated ESTs, and 69\% as unknown sequences. All the identified known genes representing broad spectrum of biological pathways were particularly linked to stress tolerance including salinity tolerance. Expression analysis of 10 known genes and 7 unknown/uncharacterized genes suggested their upregulation in the gills of prawn exposed to saline water as compared to control indicating that these are likely to be associated with salinity acclimation. Rapid amplification of cDNA ends (RACE) was used for obtaining full-length cDNA of MRSW-40 clone that was highly upregulated during salt exposure. The sequenced ESTs presented here will have potential implications for future understanding about salinity acclimation and/or tolerance of the prawn.
\end{abstract}

\section{Introduction}

The giant river prawn, Macrobrachium rosenbergii, is a commercially important shrimp widely distributed in India and neighboring countries. Euryhalinity is the ability of an aquatic organism to tolerate wide salinity variations without compromising life process. $M$. rosenbergii is a euryhaline species that can survive in a wide range of salinity conditions. $M$. rosenbergii grows in freshwater but migrates to saline water $(\sim 10 \mathrm{ppt})$ for the purposes of breeding and subsequent nursery rearing of larvae [1]. The estuarine environment is essential for the completion of larval metamorphosis and thereafter postlarvae undertake a return migration to the riverine region [2]. Migration is believed to be associated with the proc- ess of adaptability in different saline conditions. This species experiences different local microenvironments, especially salinity and temperature, during migration. As a result, they must have developed an osmoregulatory adaptive mechanism so as to survive in different environmental (saline) conditions.

Since $M$. rosenbergii migrates between brackish and fresh waters during their life cycle, this species will be suitable for studying underlining biochemical, physiological, and gene regulatory pathways. A change in gene expression has been an important component for stress management of living organisms [3-6]. However, very little information is available about variations in gene expressions related to salinity tolerance in $M$. rosenbergii. 
Gill plays a significant role in modulating ion transport either during freshwater to saline water acclimation or vice versa [6-9]. A substantial decrease in K-phosphatase activity including lowered $\alpha$-subunit protein levels of $\mathrm{Na}, \mathrm{K}$-ATPase, and a redistribution of enzyme activity into membrane fractions of different densities in salinity-acclimated shrimp (M. amazonicum) was documented [10]. Thus, gill tissues from shrimp exposed to fresh and saltwater should be one of the ideal organs for comparative genetic analysis of the molecular basis of physiological acclimatization. Most of the studies were restricted to one or small number of genes. The mechanisms of adaptability to saline water are expected to be a complex physiological process associated with branchial ion transport. It is essential to understand the complex physiological roles played by the gill.

The analysis of differential gene expression in the gill of shrimp exposed to saline water can reveal adaptive mechanisms to salinity stress. Identification of salinity-induced expression of transcripts in the gill will provide information about gill-specific adaptive response of the shrimp. Subtractive and suppressive hybridization ( $\mathrm{SSH}$ ) technique is an effective approach in identifying differentially expressed stress-induced genes in plants, fishes, and crustaceans [11$15]$, especially when prior sequence knowledge is lacking.

In the absence of prior sequence knowledge for $M$. rosenbergii, a salinity-enriched cDNA library was generated from the gill, by using SSH technique, in order to identify salinity-tolerant transcripts. It was possible to generate sequence information of several ESTs; those are likely to be linked with regulatory pathways during salt water acclimatization. A subset of forward subtracted transcripts were upregulated as examined by quantitative real-time PCR (qPCR) for differential expression in gill exposed to saltwater by comparing with those cultured in fresh water. The fulllength cDNA sequence information was generated for one of those transcripts being highly upregulated.

\section{Materials and Methods}

2.1. Salt Treatment. Adult M. rosenbergii were exposed to either salt water at $10 \mathrm{ppt}$ and $24 \mathrm{ppt}$ levels (freshwater was adjusted with sea water) or fresh water ( 0 ppt salinity) for 24 days in fiberglass-reinforced plastics (FRP) tank (3 separate tanks for $0 \mathrm{ppt}, 10 \mathrm{ppt}$, and $24 \mathrm{ppt}$ with 10 individuals in each tank) commonly being used for breeding purpose. After 24 days of treatments, gills of each individual were frozen in liquid nitrogen for total RNA extraction.

2.2. RNA Extraction. The mRNA extraction was performed as described earlier [16]. Briefly, frozen gill tissues of $M$. rosenbergii were ground in liquid nitrogen. Total RNA was extracted using the TRIzol reagent following manufacture's instructions (Invitrogen). DNase I-treated RNAs were precipitated and resuspended into diethyl pyrocarbonatetreated water. Equal amounts of total RNA from each saltwater or freshwater-treated condition (5 individuals of each group) were mixed. The quantity and quality of isolated total RNA were examined by spectrophotometric readings and denaturing formamide/agarose gel electrophoresis. PolyA tailed RNA enrichment was carried out using mRNA purification kit (Sigma, Aldrich, USA).

2.3. Construction of Subtracted cDNA Library. SSH was performed using PCR-select subtraction kit (Clontech, USA) as described [17] with slight modifications. Briefly, cDNA was reversely transcribed from $1.5 \mu \mathrm{g}$ of the mixed mRNA mentioned above using Mint cDNA synthesis Kit (Evrogen). Saltwater-treated cDNA was considered as tester and freshwater was treated as driver. Tester and driver cDNA were digested with RsaI, followed by adapter ligation to tester cDNA. Following two rounds of hybridization, primary and nested PCR amplifications were carried out for 27 and 12 cycles, respectively, to normalize and enrich the differentially expressed cDNAs. Subtractive efficiency was checked by PCR amplification $(18,23,28,33$, and 38 cycles) of the $\beta$-actin gene on subtracted and unsubtracted cDNA. The forward-subtracted secondary PCR products were purified and ligated into pGEM-T easy vector (Promega, USA) and transformed into Escherichia coli DH5 $\alpha$ competent cells and plated onto agar plates to generate a subtracted cDNA library.

2.4. Quantitative Real-Time PCR ( $q P C R)$. Quantitative realtime PCR (qPCR) for ESTs and the house-keeping genes were performed in triplicate for each cDNA sample using Light Cycler-480 SYBR Green I kit (Roche Diagnostics, Germany) in a Light Cycler 480 RT-PCR instrument (Roche Diagnostics, Germany) as described in $[16,18]$. The qPCR was performed on CDNA generated from RNA obtained from the pooled RNA samples. Relative mRNA levels of target genes were normalized to RpL8 expression for each sample, and normalized standard deviations were calculated. Primer annealing temperature for target genes and RpL8 was $58^{\circ} \mathrm{C}$. Negative control PCR containing RNA template and RpL8 primers was performed for each sample to rule out the possibility of genomic DNA contamination.

2.5. Rapid Amplification of cDNA Ends (RACEs). Since the expressions of MRSW-40 transcript were dramatically elevated to the tune of $\sim 15$-fold in saline-exposed prawn as compared to control ( 0 ppt salinity) fishes, its fulllength cDNA sequence information was generated, by $5^{\prime}-$ and $3^{\prime}$-RACE. RACE-PCR was performed to obtain the $5^{\prime}$ and $3^{\prime}$-ends of the MRSW-40 EST using Smarter RACE cDNA amplification kit (Clontech, USA) following protocol as described [16] with minor modifications. Gene-specific primers (GSP1 and GSP2) were designed from the obtained sequence data of MRSW-40 clone (Table 1). The first PCR was performed using GSP1 and Universal Primer A mix (UPM; provided with the kit) with program as follows: 5 cycles of $30 \mathrm{sec}$ at $94^{\circ} \mathrm{C}, 3 \mathrm{~min}$ at $64^{\circ} \mathrm{C}$ (for $3^{\prime}$-end and for $5^{\prime}$-end $70^{\circ} \mathrm{C}$ ) followed by 5 cycles of $30 \mathrm{sec}$ at $94^{\circ} \mathrm{C}, 30 \mathrm{sec}$ at $62^{\circ} \mathrm{C}$ (for $3^{\prime}$-end and for $5^{\prime}$-end $68^{\circ} \mathrm{C}$ ), $3 \mathrm{~min}$ at $72^{\circ} \mathrm{C}$ and finally followed by 25 cycles of $30 \mathrm{sec}$ at $94^{\circ} \mathrm{C}, 30 \mathrm{sec}$ at $60^{\circ} \mathrm{C}$ (for $3^{\prime}$-end and for $5^{\prime}$-end $66^{\circ} \mathrm{C}$ ), and $3 \mathrm{~min}$ at $72^{\circ} \mathrm{C}$. Then, the second PCR was performed using GSP2 and Nested Universal Primer A (NUP; provided with the 
TABLE 1: The gene-specific primers (GSP) designed for RACE PCR for clone MRSW-40.

\begin{tabular}{llcc}
\hline RACE primers & Sequence & Size $(\mathrm{bp})$ & $T_{m}$ \\
\hline GSP1 (5'-RACE) & GGCGTCTGTGGCATCATCAGACCCAGTC & 28 & $66.0^{\circ} \mathrm{C}$ \\
GSP2 (5'-RACE) & GGAGCATACGGAATGACCGTGATGG & 25 & $61.3^{\circ} \mathrm{C}$ \\
GSP1 (3'-RACE) & CCATCCTGATGATGACACACAG & 22 & $55.4^{\circ} \mathrm{C}$ \\
GSP2 (3'-RACE) & TCAAATCCCCTCACCACACTGC & 22 & $59.4^{\circ} \mathrm{C}$ \\
\hline
\end{tabular}

kit) with program as follows: 25 cycles of $30 \mathrm{sec}$ at $94^{\circ} \mathrm{C}$, $30 \mathrm{sec}$ at $60^{\circ} \mathrm{C}$ (for $3^{\prime}$-end) and $66^{\circ} \mathrm{C}$ (for $5^{\prime}$-end), and $3 \mathrm{~min}$ at $72^{\circ} \mathrm{C}$. PCR products were separated on a $1.5 \%$ agarose gel, and desired bands were excised for purification using gel extraction kit (USB, USA). The purified fragments were cloned into pGEMT easy vector (Promega, USA) and then transformed into chemically competent $\mathrm{DH} 5 \alpha$ cells. The positive inserts were sequenced using an automated ABI 310 genetic analyzer (Perkin-Elmer Applied Bios system). The sequences were verified using the BLASTN program (http://www.ncbi.nlm.nih.gov/blast). The amino acid sequence was deduced by Expasy translate tool (http://expasy.org/tools/dna.html) and verified using BLASTP at the same site.

\section{Results}

3.1. Assembly and Characterization of M. rosenbergii ESTs Generated by SSH. To identify the genes involved in adaptability to saline water, a forward-subtracted cDNA library was constructed from the pooled mRNA extracted from the gill of $M$. rosenbergii exposed to salt water (10 and $24 \mathrm{ppt}$ ) representing tester cDNA and freshwater exposure as driver. The enriched transcripts generated by SSH are expected to be the representation of salinity-induced transcripts. Subtraction efficiency was evaluated by comparing the amplified housekeeping $\beta$-actin gene with the templates of subtractive and unsubtractive PCR products of second round (Figure 1(a)). In the subtracted library, 33 cycles were necessary to detect the amplified band of $\beta$-actin gene compared with 23 cycles in the unsubtracted library. A substantial reduction in $\beta$-actin gene cDNA to subtracted testis cDNA indicated that a large number of constitutive genes were removed effectively, and salinity-specific genes were enriched successfully.

After subtraction, the cDNA fragments were cloned, and a total of 162 randomly picked clones contained inserts, ranging from $90 \mathrm{bp}$ to $850 \mathrm{bp}$, and were considered for sequencing. Of the 162 clones sequenced, 127 nonrepeated, good-quality sequences were aligned to those in the GenBank databases. Ten clones belonged to the ribosomal proteins. Figure 1(b) shows the BLAST results for the rest of 117 gene transcripts identified and submitted to GenBank (GenBank Accession nos. JK502144-JK502260). Sixty-nine percent (81 clones) of the unique sequences exhibited no significant homology to any previously identified genes (termed unknown). About 22\% (26 clones) of putative transcripts showed significant $(>65 \%)$ sequence homology with vertebrate genes (termed known) as shown in Table 2, while 10 EST fragments (9\%) showed homology only with

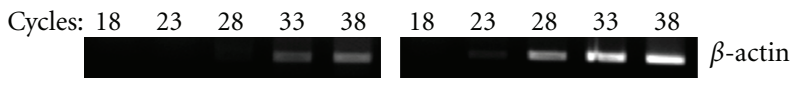

(a)

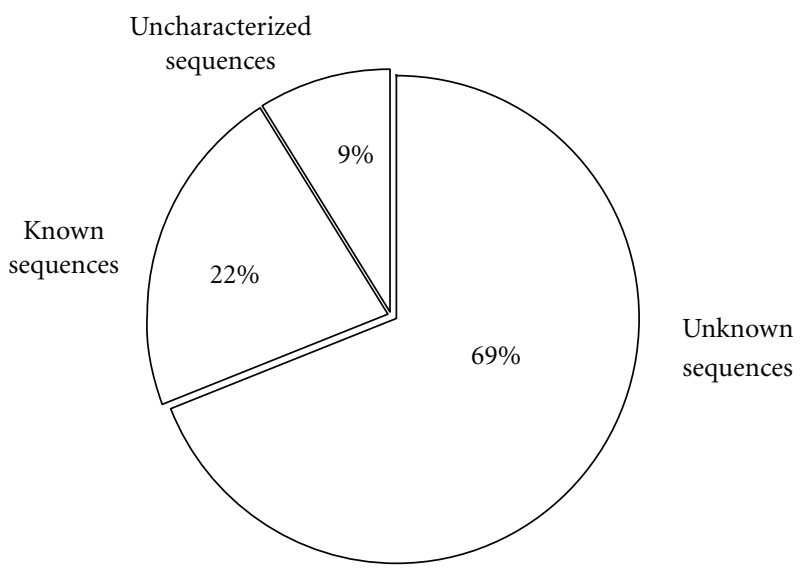

(b)

FIGURE 1: Subtraction efficiency verification and characterization of ESTs. (a) Subtraction efficiency was estimated by polymerase chain reaction (PCR) amplification of $\beta$-actin gene on subtracted and unsubtracted cDNA libraries. The number of PCR cycles is indicated above each lane. (b) EST classification represented in subtracted library based on sequence analysis of 117 nonredundant inserts. Known sequences exhibit significant homology with known genes. Uncharacterized sequences were homologous to unannotated EST sequences. Sequences with no significant match were called unknown sequences.

unannotated ESTs (termed uncharacterized) as shown in Table 3. Most of the uncharacterized ESTs matched with crustacean ESTs generated from various organs including gill (Table 3). All the clones of known category matched with stress-induced genes including 12 clones linked to salt tolerances for fish and shellfish species (Table 2 ). This further validated the assertion that efficient enrichment of transcripts for salinity tolerance was achieved. Functionally known transcripts represented broad spectrum of biological pathways such as energy metabolism, signal transduction, transcription factors, and transport facilitators.

3.2. Relative Expression Pattern of Known and Unknown ESTs in the Gills of M. rosenbergii Exposed to Saline Water. To validate the outcomes of SSH-mediated enrichment of salinity-induced transcripts, qPCR was performed to quantify the abundance of selected ESTs. Differential quantifications were examined for few known and unknown transcripts in the gills of $M$. rosenbergii exposed to seawater (adjusted to $10 \mathrm{ppt}$ salinity) and freshwater ( $0 \mathrm{ppt}$ salinity). 


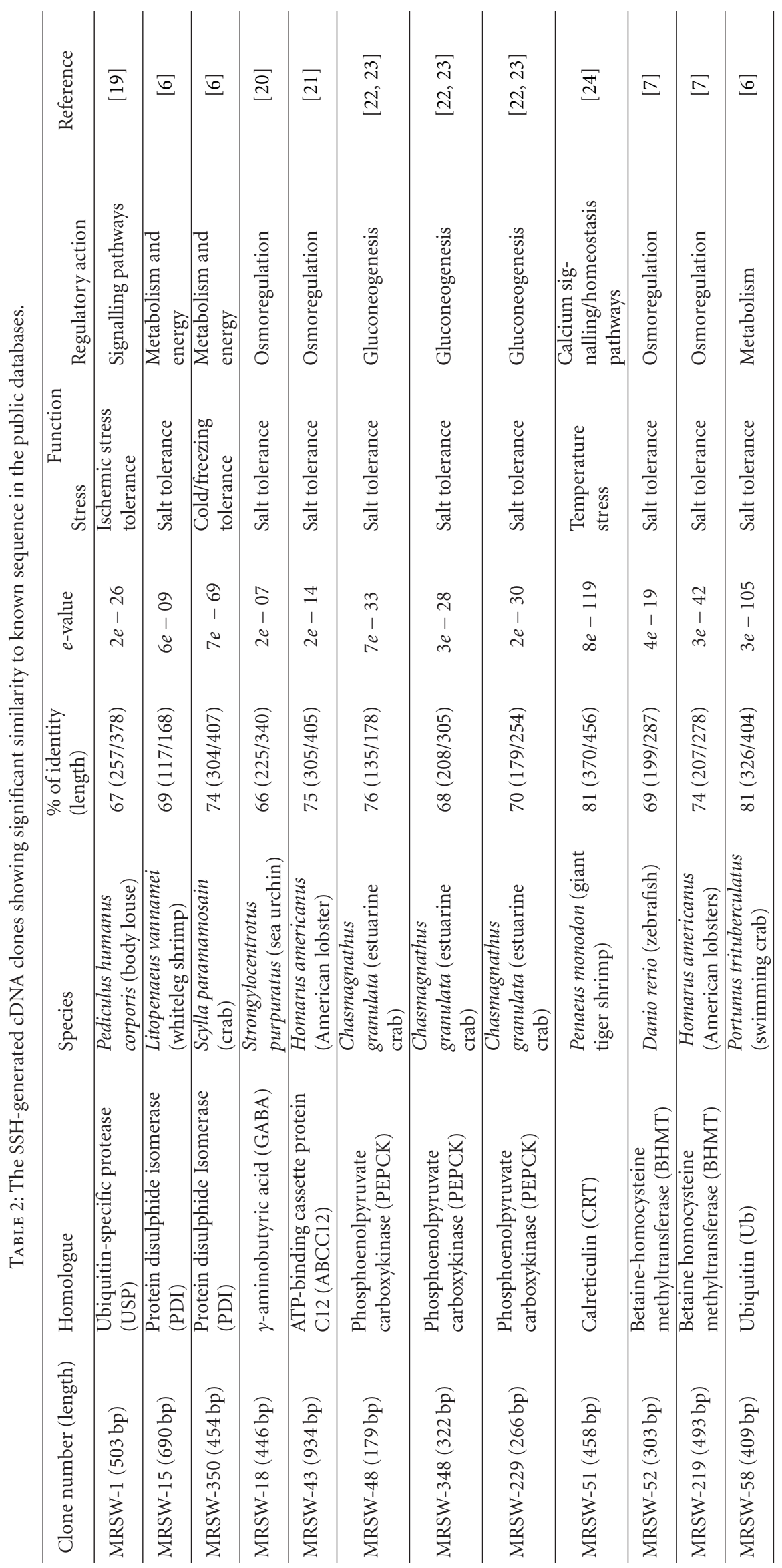




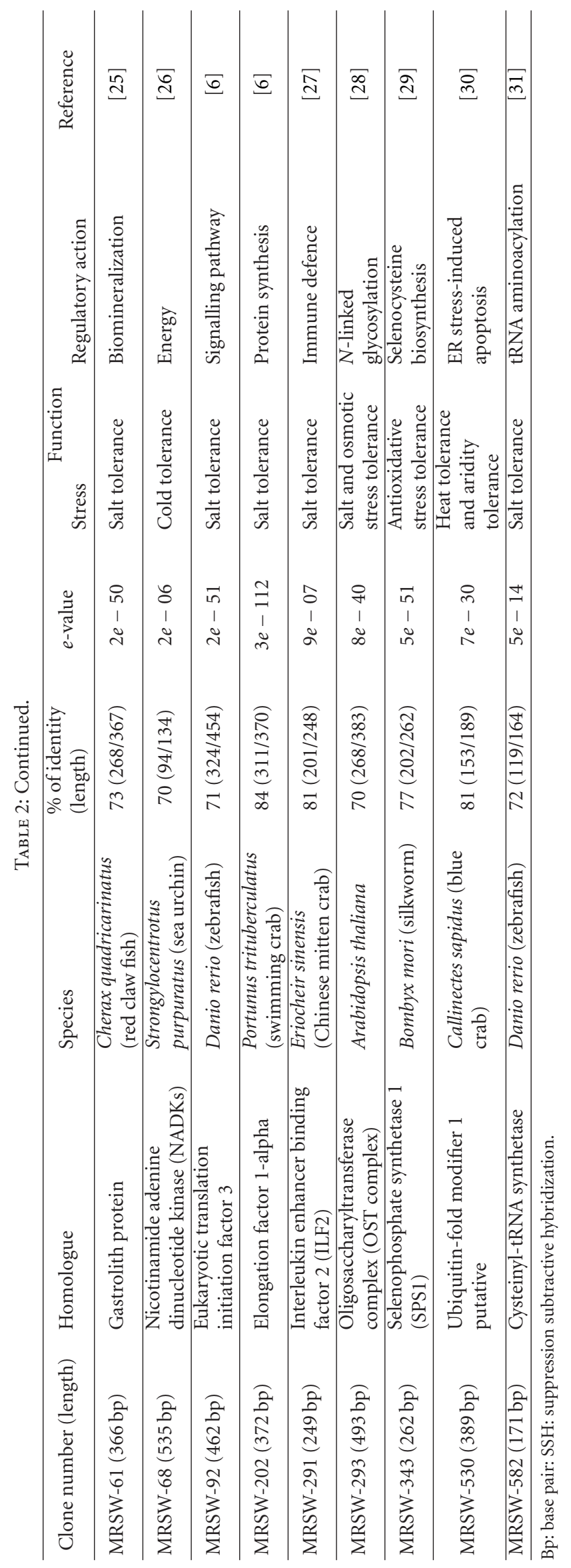




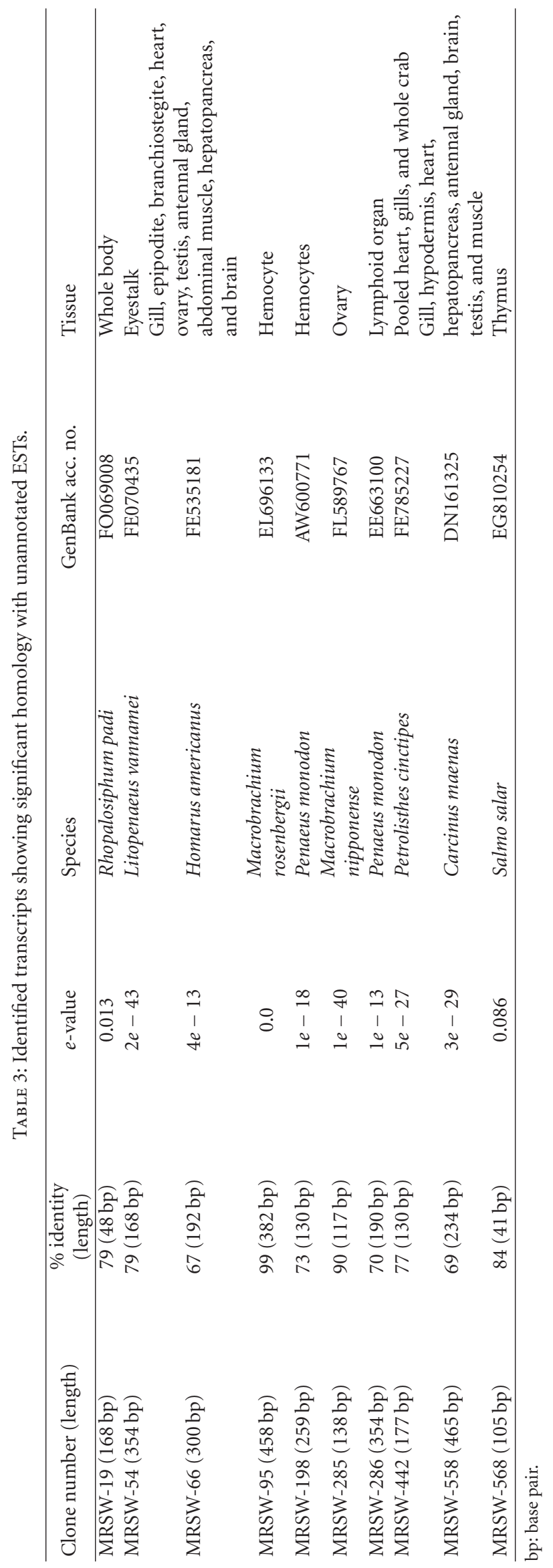


Seawater at $10 \mathrm{ppt}$ salinity was chosen because hatcherybased breeding for $M$. rosenbergii in a mass scale is generally performed at around $10 \mathrm{ppt}$ level. The known EST clones were selected based on earlier evidence associated with salinity-stress tolerance in other fish/crustacean and quality of sequence generated by SSH. Pooled mRNAs from the gills of $\geq 5$ individuals from each experimental condition were selected for cDNA preparation.

Evaluation of the best reference genes for qPCR is essential. Expression stability for house-keeping genes in the larvae, postlarvae, and gill of adult $M$. rosenbergii exposed to salt water and freshwater was analyzed using geNorm rankings as described $[16,32]$. In this study, the mRNA quantity levels for GAPDH, RpL8, RpL18, $\beta$-actin, and Elf $\alpha$ house-keeping genes were tested. Among these, expression of RpL8 was identified as the most stable (data not shown) consistent with earlier findings $[6,11]$, and hence, the qPCR data was normalized with RpL8.

Figure 2 shows the fold change obtained between saltexposed and control samples. As expected, the relatively elevated levels of mRNA expressions ( $\geq 1.5$-fold) were detected in salt-treated gill samples more than control with 9 interested known genes such as ubiquitin-specific protease (USP, MRSW-1), protein disulfide isomerase (PDI, MRSW350), $\gamma$-aminobutyric acid (GABA, MRSW-18), ATP-binding cassette protein $\mathrm{C} 12$ (ABCC12, MRSW-43), calreticulin (CRT, MRSW-51), interleukin enhancer binding factor 2 (ILF2, MRSW-291), oligosaccharyltransferase complex (OST complex, MRSW-293), selenophosphate synthetase 1 (SPS1, MRSW-343), and phosphoenolpyruvate carboxykinase (PEPCK, MRSW-348). Heat shock protein 70 (HSP70) was known to be upregulated in brown trout and rainbow trout while migrated to saline water $[5,33]$, but it possibly was not picked up as a clone in our study. HSP70 was taken as a positive control for our qPCR analysis. As expected, HSP70 expression was up-regulated in the gill of saline-treated prawns (Figure 2). These findings suggested their physiological significance with regard to osmoregulatory adaptive mechanism in diverse species including $M$. rosenbergii.

Of the unknown EST clones, the abundance of expressions for MRSW-40 and MRSW-559 clones was maximum in the tune of $\sim 15$ - and $\sim 26$-fold, respectively, in salttreated gills (Figure 2). The significantly higher level of expression for MRSW-195 was documented due to salt exposure. Salt exposure also caused increased levels $(\geq 1.5$ folds) of MRSW-38, MRSW-54, MRSW-98, and MRSW-532 mRNAs expressions in the gill. Thus, 7 unknown clones, being up-regulated in the gills of $M$. rosenbergii cultured in saline water, could be considered as novel ESTs that are most likely to be linked with salinity-stress tolerance.

3.3. Generation of Full-Length cDNA Sequence for MRSW40 EST. The RACE-derived full-length cDNA sequence of MRSW-40 transcript contained an open reading frame (ORF) of 1125 nucleotides translatable to 374 aa with an ATG (M) start codon and a TGA stop codon (Figure 3). The first start codon was within the consensus sequence of GTTATGG fulfilling the Kozak criteria (A/GNNATGG) [34]. The sequence consisted of $623 \mathrm{bp} 5^{\prime}$-flanking region,

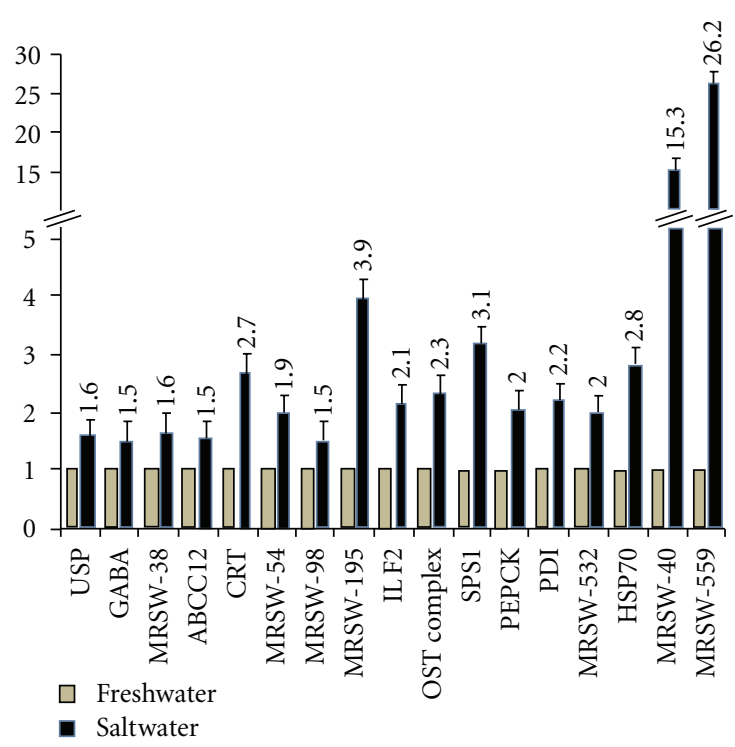

FIGURE 2: The expression profiles of selected ESTs enriched from SSH cDNA library by quantitative real-time polymerase chain reaction (qPCR). The qPCR data for all ESTs was normalized with RPL8 as reference gene. The qPCR data shows the relative gene expression levels in the salt water-stressed gill of $M$. rosenbergii over unstressed gill (default setting as 1 in each case). The clones MRSW-40 and MRSW-559 were significantly up-regulated in the tune of about 15- and 26-fold, respectively. The rest of the clones were up-regulated in the tune of $\geq 1.5$-fold. The numbers on top of each bar for salt water-treated gill represent fold changes in expressions relative to the expressions in freshwater-treated gill. The data represents the average of three independent (each in duplicate) experiments. USP, ubiquitin-specific protease; GABA, $\gamma$-aminobutyric acid; ABCC12, ATP-binding cassette protein $\mathrm{C} 12$; CRT, calreticulin; ILF2, interleukin enhancer binding factor 2; OST complex, oligosaccharyltransferase complex; SPS1, selenophosphate synthetase 1; PEPCK, phosphoenolpyruvate carboxykinase; PDI, protein disulphide isomerase; HSP70, heat shock protein 70.

relative to start codon, and $311 \mathrm{bp} 3^{\prime}$-UTR. The poly-A tail was identified within $3^{\prime}$-untranslated tail. A schematic representation of deduced amino acid (aa) for clone MRSW40 is shown in Figure 3. The aa sequence derived from the clone MRSW40 was analyzed with the help of Expasy tool (www.expasy.org/tools/dna.html). Two important domains such as Glutamine- and Serine-rich corresponding to 16 to 37 aa and 89 to 182 aa, respectively, were identified. Thus, it was possible to identify and characterize full-length sequence information of one possible novel cDNA, whose expression levels are affected by salinity exposure.

\section{Discussion}

The present study is the first attempt to explore gene expression in the gill of commercially important $M$. rosenbergii exposed to saline water. In euryhaline species, adaptability to saline water is likely to be associated with complex trait, which is controlled and regulated by many genes. Because SSH works efficiently, especially in the absence of prior sequence information, we utilized this technique for 
-623 bp GAGCTCCCAAAACAACTCTTCGC GT T GCTCCTGCGGGGTCT GCACCAAACGGCAAT GGGCCT AAGGT GGACCCT AACGACCCC CAGGTCCGCAAAT T AGTCT ACAATGCGT ACAGGGAGAT GCTT AGCAAGAAGCACGCCGAT GCCAAT GT GATT GT GGACAGCGCCCCGCAGGAACTT GT GAT CAAGGACCACGGCGTCGGT TCCCGCGTCGAAAGCATGATGCGGCAGCAAAGCGAGTT AAAGGGAGGGAAT ATGAATGCT GGCGGACCACCTCCACCCCCACCACCACCACCTAT CCCAGGCATCATCCCAACTTCAAGA CT TCCTTCAGAGATCCCCGCTCGCCCAGCCAT GAAT ACCCCACCTGCT GCCTTGGCCAGT GCTAT GAT GAAGGACAAGAAGCCCTTCACAT ACACCCCAGGAGGACTCGAT TT GT CCGAA ATTAGTAACCAGAATGGTGACCAAGCAGATATTGGT GCCCCTGTCCAATCTCGGTCTTTC AGAGCGCT GCAGAAT GTTTTGGACCACCAAGACAAT GCT GT ACGAATATCAGCTAATACT GATGAAGCCCGGGCAAGAGGT GGACACT T TCCTAAAGAGAATTTT AATTCAGGTTAAAAA (+1 start site)

at ggaacctcaagggcct at ccat at cccaaacatgaagcatgctcagcagcctagccag 60

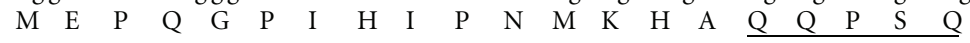

$\mathrm{t}$ accagactcaacccaaccagt $\mathrm{t} c \mathrm{aacagcagcatgtt} \mathrm{t}$ at caaca acagtccggtgt a 120

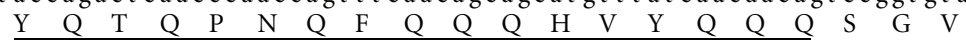
a at at at gcaggcaat acagagcaggcagttcccgagccgaagagtacatgggcggc 180

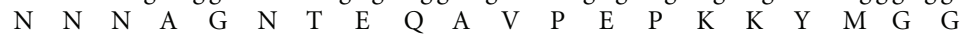
a a t t c cat cacggtcat tccgt at gctccaagccatgactgggtctgat gat gecaca 240 $\begin{array}{lllllllllllllllllllll}N & I & P & S & R & S & F & R & M & L & Q & A & M & T & G & S & D & D & A & T\end{array}$ gacgccggaga actgccaat act tetgat ct tccagccatcctgat gat gacacacag 300

$\begin{array}{llllllllllllllllllll}D & A & G & E & P & A & N & T & S & E & S & S & S & H & P & D & D & D & T & Q\end{array}$

cat acagccaat caa t cccetcaccacactgcaa t cctcaat tcctaactcaccctct 360

$\begin{array}{llllllllllllllllllll}\mathrm{H} & \mathrm{T} & \mathrm{A} & \mathrm{N} & \mathrm{Q} & \mathrm{I} & \mathrm{P} & \mathrm{S} & \mathrm{P} & \mathrm{H} & \mathrm{C} & \mathrm{K} & \mathrm{S} & \mathrm{S} & \mathrm{I} & \mathrm{P} & \mathrm{N} & \mathrm{S} & \mathrm{P} & \mathrm{S}\end{array}$

t ccctcact t ctctacacatgacat t ct t ctgacagt ggat atcatcccettcaaa 420

$\begin{array}{lllllllllllllllllllllllllll}\mathrm{S} & \mathrm{L} & \mathrm{T} & \mathrm{S} & \mathrm{P} & \mathrm{T} & \mathrm{H} & \mathrm{D} & \mathrm{I} & \mathrm{S} & \mathrm{S} & \mathrm{D} & \mathrm{S} & \mathrm{G} & \mathrm{I} & \mathrm{S} & \mathrm{S} & \mathrm{P} & \mathrm{S} & \mathrm{K}\end{array}$

at tggt tct t acacaagt ggt a agat agt a aggt t atcggcatgtgtctcccaagt c 480

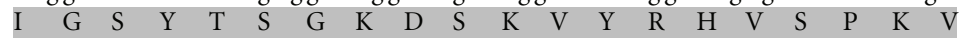

act t caatggctctaagagtgggt gt gaat tcagccct a a caat tcaagggaacca 540

$\begin{array}{lllllllllllllllllllll}T & F & N & G & S & K & E & W & V & V & N & S & A & L & S & N & S & R & E & P\end{array}$

tcatcacct act tggaaacagtgaatccccatcct at t ccaagcaccgtaccagt t a a 600

$\begin{array}{llllllllllllllllllll}\mathrm{S} & \mathrm{S} & \mathrm{P} & \mathrm{T} & \mathrm{W} & \mathrm{K} & \mathrm{T} & \mathrm{V} & \mathrm{N} & \mathrm{P} & \mathrm{H} & \mathrm{P} & \mathrm{I} & \mathrm{P} & \mathrm{S} & \mathrm{T} & \mathrm{V} & \mathrm{P} & \mathrm{V} & \mathrm{N}\end{array}$

aggcct tcacctgat tctccttgtcaggat agt ccaagactcgtgaactcacagggaa 660

$\begin{array}{llllllllllllllllllll}R & P & S & P & D & S & P & C & Q & D & S & P & R & L & V & K & L & T & G & E\end{array}$

gaatcagccagtcctagccctcaa at cagagtgttgaaaaggt at gagggggacac 720

$\begin{array}{lllllllllllllllllllll}E & S & A & S & P & S & P & Q & N & Q & S & V & E & K & R & Y & E & G & G & H\end{array}$

at $t c c t$ ctcgtgtct t agacat ct acaagctgaat act ct t ctggtgact ct a a gaa 780

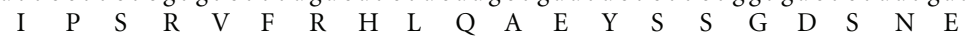

ct tcatgagccaaget gcaat t gat tcaagagtcct tcacct tgt t a a gegt agt 840

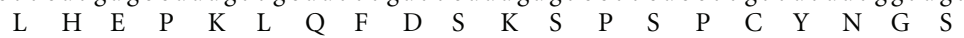

cct at tccaccaagagt actgaa tcct t acagaatgacact a t a a a g t t t gat tca 900

$\begin{array}{llllllllllllllllllll}\mathrm{P} & \mathrm{I} & \mathrm{P} & \mathrm{P} & \mathrm{R} & \mathrm{V} & \mathrm{L} & \mathrm{K} & \mathrm{S} & \mathrm{L} & \mathrm{Q} & \mathrm{N} & \mathrm{D} & \mathrm{T} & \mathrm{N} & \mathrm{K} & \mathrm{S} & \mathrm{F} & \mathrm{D} & \mathrm{S}\end{array}$

gat at a a t gatggggaa a c ct t caagagct tcaagt tct t agctgaact agatgaa 960

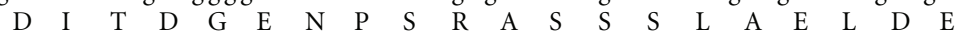

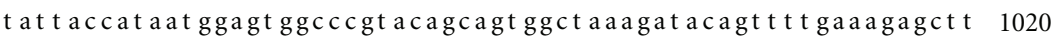

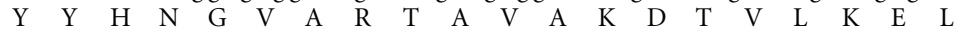

actgctcagt t gaccagactggagt a aggaaa acct aagctgcccetggt agagt t 1080

$\begin{array}{lllllllllllllllllllll}\mathrm{T} & \mathrm{A} & \mathrm{Q} & \mathrm{F} & \mathrm{D} & \mathrm{Q} & \mathrm{T} & \mathrm{G} & \mathrm{V} & \mathrm{R} & \mathrm{K} & \mathrm{K} & \mathrm{P} & \mathrm{K} & \mathrm{L} & \mathrm{P} & \mathrm{L} & \mathrm{V} & \mathrm{E} & \mathrm{F}\end{array}$

$\mathrm{t} c \mathrm{cgct}$ act $\mathrm{t}$ a cagt $\mathrm{cac}$ agt at gat act ccggaggat gat t $\mathrm{tag}$ AACAACAGTATTCTC 1140

$\begin{array}{llllllllllllllllllllll}\mathrm{S} & \mathrm{A} & \mathrm{T} & \mathrm{Y} & \mathrm{S} & \mathrm{H} & \mathrm{S} & \mathrm{M} & \mathrm{I} & \mathrm{L} & \mathrm{R} & \mathrm{R} & \mathrm{M} & \mathrm{I} & -\end{array}$

AAACAGATAAAGACACCTTAAAACCAGAGCCAGAAGAAACAACAGTATTCTCTAACTCAG 1200 GATTTGGTTATAGAGGAGCAAAAGTTCCCTCCCCTTCATTCAAATTTTTGCAAAGTCAGT 1260 ACAGCCAAGAGCAAGAACAGGTAAAACCTAGTGAATCTGAACCTTCATATCCTGATACCA 1320

CAGAGTTAAAGGAGGAACCTCTTCCATATAAAGGAT GTCGTGTCCCTGGCCGTACTTTTA 1380

GGTCTCTTCAAGACAATGTTACAACACATCCTTCAGTTTTAAAGCCAAAAAAAAAAAAAA 1440

AAAAAAAAAAA

1451

FIgURE 3: The full-length cDNA sequence of MRSW-40 EST derived through RACE and the deduced amino acid sequence. The ORF is shown in small letters which encode 374 amino acids. The in-frame stop codon in the cds is marked within a box. The $5^{\prime}$ - and $3^{\prime}$-UTR are shown in capital letters. The identified glutamine-rich domain is underlined, and the serine-rich motif is shaded within gray box.

efficiently enriching a subset of genes that are up-regulated by salinity stress in the gill of $M$. rosenbergii. We have identified 26 ESTs encoding regulatory proteins associated with biological processes of salinity stress management.

The encoded phosphoenolpyruvate carboxykinase (PEPCK), the key gluconeogenic enzymes associated with energy-requiring metabolic pathway, was enriched in the cDNA library. The elevated transcriptional level of PEPCK during hyperosmotic stress was documented earlier in magur (Clarias batrachus) and a euryhaline crab (Chasmagnathus granulate) $[22,23,35]$. Protein disulfide isomerase (PDI) is also involved in metabolic pathway, and its upregulation in the gill of saline-water-exposed prawns indicated that high-energy-demanding metabolic pathways are involved in salinity acclimation. Additionally, components of signaling pathways, transporters, and transcriptional regulators such as ubiquitin-specific protease (USP), $\gamma$-aminobutyric acid (GABA), oligosaccharyltransferase (OST) complex, calreticulin, and elongation factor 1 were up-regulated during saline exposure demonstrating that gill is actively involved 
in many cellular functions for transcriptional regulation of metabolism and maintaining homeostasis for saline water adaptability. USP was reported to be involved in managing ischemic stress tolerance [19]. Here, we provide evidence that it is involved in salinity stress tolerance. Calreticulin, located in endoplasmic reticulum (ER) lumen, has been functionally linked with signal transduction and homeostasis of $\mathrm{Ca}^{2+}$ including acting as a protein-folding chaperone. It is involved in many biological processes of growth, reproduction, molting [36], and stress responses of temperature, oxidative, and heavy metal contamination [24]. From this study, it is evidenced that it could also be used as a biomarker for salinity stress responses. Interleukin enhancer binding factor 2 (ILF2), a known transcriptional regulator for T-cellmediated immune defense against biotic stress caused by infection [27], is up-regulated and hence might be involved in physiological process of euryhalinity. ATP-binding cassette (ABC) transporters form one of the largest protein families. $\mathrm{ABC}$ containing proteins participates in regulating ion channels in the plasma membrane, especially ATP-sensitive potassium channels [21], and thus the ABC transcript identified in our study is most likely to be associated with osmoregulatory pathway. ABC protein $\mathrm{C} 12$ (ABCC12), a variant belonging to $\mathrm{ABC}$ gene family, has been cloned and characterized in mouse and human [37]. Its expression in various organs with highest in testis was documented. From this study, it appears that ABCC12 plays a significant role in salinity adaptation in the gill. However, its exact participatory role in salinity tolerance remains to elucidate. OST complex is a transporter that transfers the core oligosaccharide via $N$-linkage to an Asn residue of nascent peptide for downstream-regulated maintenance of homeostasis during salinity/osmotic stress [28]. An alternative pathway of selenocysteine (Sec) biosynthesis mediated by selenophosphate 1 (SPS1) protein exists only in aquatic arthropods including insects, but not in other eukaryotes, for oxidative stress tolerance [29]. In this study, SPS1 was found to be involved in salinity stress tolerance.

Of the 81 genes of unknown functions obtained from the SSH library, 7 selected transcripts (MRSW-38, MRSW40, MRSW-54, MRSW-98, MRSW-195, MRSW-532, and MRSW-559) were up-regulated ( $\geq 1.5$-folds) in the gill of saline-treated prawns. These transcripts are of particular interest because of their likely potential functions with salinity stress tolerances. Among these, since the expressions of MRSW-40 and MRSW-559 transcripts were dramatically elevated in the tune of more than 15 -fold in saline-exposed prawn as compared to control (0 ppt salinity), the full-length cDNA sequence information was generated for MRSW-40 EST to characterize and ascertain its functional relevance. The deduced 374 amino acid sequence of it contained two important domains, that is, glutamine- and serine-rich motifs. The regulatory roles of these two domains present in other proteins were associated with stress tolerance [38, 39]. It is believed that variable repeat-containing ORFs are associated with transcriptional regulation. Polymorphic proteins containing repeat sequences confer adaptive survival of the cell in the extreme environmental conditions. The presence of relatively longer $5^{\prime}$-UTR is most likely to be associated with regulatory noncoding regions. In this study, the knowledge gained from the full-length sequence for MRSW-40 would be of great help, in future, in dissecting regulatory role being played by it during salinity stress induction.

In conclusion, an up-regulated SSH library was constructed successfully from the gills of $M$. rosenbergii exposed to saline water. The ESTs screened from the library encode various molecules potentially associated with different biological processes, which are involved in cellular metabolic processes, signal transduction and biological regulation, response to stimuli, and other functions and unknown functions. The information generated from this study is expected to provide new insights into the salinity-mediated stress tolerance mechanisms of $M$. rosenbergii. Future studies could be undertaken to functionally validate these ESTs in vivo and to uncover physiological significance in response to salinity tolerance.

\section{Acknowledgment}

This project work was funded by Indian Council of Agricultural Research (ICAR) under National Agricultural Innovation Project (NAIP) Scheme, Government of India, India.

\section{References}

[1] E. John, "Physico-chemical studies of river Pumba and distribution of prawn, Macrobrachium rosenbergii," Journal of Environmental Biology, vol. 30, no. 5, pp. 709-712, 2009.

[2] M. B. New, "Farming freshwater prawns. a manula for the culture of the giant river prawn (Macrobrachium rosenbergii)," FAO Fisheries Technical Paper 428, 212, 2002.

[3] J. Shaterian, F. Georges, A. Hussain, D. Waterer, H. De Jong, and K. K. Tanino, "Root to shoot communication and abscisic acid in calreticulin $(C R)$ gene expression and salt-stress tolerance in grafted diploid potato clones," Environmental and Experimental Botany, vol. 53, no. 3, pp. 323-332, 2005.

[4] A. Pandit, V. Rai, S. Bal et al., "Combining QTL mapping and transcriptome profiling of bulked RILs for identification of functional polymorphism for salt tolerance genes in rice (Oryza sativa L.)," Molecular Genetics and Genomics, vol. 284, no. 2, pp. 121-136, 2010.

[5] P. F. Larsen, E. E. Nielsen, A. Koed, D. S. Thomsen, P. A. Olsvik, and V. Loeschcke, "Interpopulation differences in expression of candidate genes for salinity tolerance in winter migrating anadromous brown trout (Salmo trutta L.)," BMC Genetics, vol. 9, article 12, 2008.

[6] Q. Xu and Y. Liu, "Gene expression profiles of the swimming crab Portunus trituberculatus exposed to salinity stress," Marine Biology, vol. 158, no. 10, pp. 2161-2172, 2011.

[7] X. J. Lu, J. Chen, Z. A. Huang, Y. H. Shi, and F. Wang, "Proteomic analysis on the alteration of protein expression in gills of ayu (Plecoglossus altivelis) associated with salinity change," Comparative Biochemistry and Physiology D, vol. 5, no. 3, pp. 185-189, 2010.

[8] G. R. Scott and P. M. Schulte, "Intraspecific variation in gene expression after seawater transfer in gills of the euryhaline killifish Fundulus heteroclitus," Comparative Biochemistry and Physiology A, vol. 141, no. 2, pp. 176-182, 2005. 
[9] W. S. Marshall, " $\mathrm{Na}^{+}, \mathrm{Cl}^{-}, \mathrm{Ca}^{2+}$ and $\mathrm{Zn}^{2+}$ transport by fish gills: retrospective review and prospective synthesis," Journal of Experimental Zoology, vol. 293, no. 3, pp. 264-283, 2002.

[10] N. M. Belli, R. O. Faleiros, K. C. S. Firmino et al., "Na,KATPase activity and epithelial interfaces in gills of the freshwater shrimp Macrobrachium amazonicum (Decapoda, Palaemonidae)," Comparative Biochemistry and Physiology A, vol. 152, no. 3, pp. 431-439, 2009.

[11] D. Cottin, B. Shillito, T. Chertemps, A. Tanguy, N. Léger, and J. Ravaux, "Identification of differentially expressed genes in the hydrothermal vent shrimp Rimicaris exoculata exposed to heat stress," Marine Genomics, vol. 3, no. 2, pp. 71-78, 2010.

[12] S. Daldoul, S. Guillaumie, G. M. Reustle et al., "Isolation and expression analysis of salt induced genes from contrasting grapevine (Vitis vinifera L.) cultivars," Plant Science, vol. 179, no. 5, pp. 489-498, 2010.

[13] Y. Wu, Q. Wang, Y. Ma, and C. Chu, "Isolation and expression analysis of salt up-regulated ESTs in upland rice using PCRbased subtractive suppression hybridization method," Plant Science, vol. 168, no. 3, pp. 847-853, 2005.

[14] T. S. Hori, A. K. Gamperl, L. O. B. Afonso et al., "Heatshock responsive genes identified and validated in Atlantic cod (Gadus morhua) liver, head kidney and skeletal muscle using genomic techniques," BMC Genomics, vol. 11, no. 1, article 72, 2010.

[15] D. Kültz, D. Fiol, N. Valkova, S. Gomez-Jimenez, S. Y. Chan, and J. Lee, "Functional genomics and proteomics of the cellular osmotic stress response in "non-model" organisms," Journal of Experimental Biology, vol. 210, no. 9, pp. 1593-1601, 2007.

[16] C. Mohapatra, H. K. Barman, R. P. Panda et al., "Cloning of cDNA and prediction of peptide structure of Plzf expressed in the spermatogonial cells of Labeo rohita," Marine Genomics, vol. 3, no. 3-4, pp. 157-163, 2010.

[17] H. K. Barman, R. P. Panda, C. Mohapatra, A. Swain, and A. E. Eknath, "Identification of genes preferentially expressed in testis and spermatogonial cells of Labeo rohita by subtractive and suppressive hybridization," Aquaculture Research, vol. 42, no. 8, pp. 1196-1205, 2011.

[18] R. P. Panda, H. K. Barman, and C. Mohapatra, "Isolation of enriched carp spermatogonial stem cells from Labeo rohita testis for in vitro propagation," Theriogenology, vol. 76, no. 2, pp. 241-251, 2011.

[19] O. Zolk, C. Schenke, and A. Sarikas, "The ubiquitinproteasome system: focus on the heart," Cardiovascular Research, vol. 70, no. 3, pp. 410-421, 2006.

[20] J. M. Barbosa, R. D. Locy, T. W. Barger, N. K. Singh, and J. H. Cherry, "GABA increases the rate of nitrate uptake and utilization in Arabidopsis roots," Plant Biology, vol. 83, pp. 5363, 2000.

[21] E. K. Lee, M. Kwon, J. H. Ko et al., "Multidrug resistancerelated protein that functions in salt tolerance," Plant Physiology, vol. 134, no. 1, pp. 528-538, 2004.

[22] V. Schein, Y. Waché, R. Etges, L. C. Kucharski, A. van Wormhoudt, and R. S. M. Da Silva, "Effect of hyperosmotic shock on phosphoenolpyruvate carboxykinase gene expression and gluconeogenic activity in the crab muscle," FEBS Letters, vol. 561, no. 1-3, pp. 202-206, 2004.

[23] V. Schein, A. L. Fernandes Chittó, R. Etges, L. C. Kucharski, A. van Wormhoudt, and R. S.M. Da Silva, "Effect of hyper or hypo-osmotic conditions on neutral amino acid uptake and oxidation in tissues of the crab Chasmagnathus granulata,"
Comparative Biochemistry and Physiology B, vol. 140, no. 4, pp. 561-567, 2005.

[24] V. Visudtiphole, A. Watthanasurorot, S. Klinbunga, P. Menasveta, and K. Kirtikara, "Molecular characterization of Calreticulin: a biomarker for temperature stress responses of the giant tiger shrimp Penaeus monodon," Aquaculture, vol. 308, no. 1, pp. S100-S108, 2010.

[25] A. Shechter, L. Glazer, S. Cheled et al., "A gastrolith protein serving a dual role in the formation of an amorphous mineral containing extracellular matrix," Proceedings of the National Academy of Sciences of the United States of America, vol. 105, no. 20, pp. 7129-7134, 2008.

[26] J. M. Ruiz, E. Sanchez, P. C. Garcia, L. R. Lopez-Lefebre, R. M. Rivero, and L. Romero, "Proline metabolism and NAD kinase activity in greenbean plants subjected to cold-shock," Phytochemistry, vol. 59, no. 5, pp. 473-478, 2002.

[27] J. Yang, L. Wang, M. Huang et al., "An interleukin-2 enhancer binding factor 2 homolog involved in immune response from Chinese mitten crab Eriocheir sinensis," Fish and Shellfish Immunology, vol. 30, no. 6, pp. 1303-1309, 2011.

[28] H. Koiwa, F. Li, M. G. McCully et al., “The STT3a subunit isoform of the arabidopsis oligosaccharyltransferase controls adaptive responses to salt/osmotic stress," Plant Cell, vol. 15, no. 10, pp. 2273-2284, 2003.

[29] A. V. Lobanov, D. L. Hatfield, and V. N. Gladyshev, "Selenoproteinless animals: selenophosphate synthetase SPS1 functions in a pathway unrelated to selenocysteine biosynthesis," Protein Science, vol. 17, no. 1, pp. 176-182, 2008.

[30] K. Lemaire, R. F. Moura, M. Granvik et al., "Ubiquitin fold modifier 1 (UFM1) and its target UFBP1 protect pancreatic beta cells from ER stress-induced apoptosis," PLoS ONE, vol. 6, no. 4, pp. 1-14, 2011.

[31] H. Jakubowski, "Editing function of Escherichia coli cysteinyltRNA synthetase: cyclization of cysteine to cysteine thiolactone," Nucleic Acids Research, vol. 22, no. 7, pp. 1155-1160, 1994.

[32] J. Vandesompele, K. De Preter, F. Pattyn et al., "Accurate normalization of real-time quantitative RT-PCR data by geometric averaging of multiple internal control genes," Genome Biology, vol. 3, no. 7, article RESEARCH0034, 2002.

[33] C. J. Niu, J. L. Rummer, C. J. Brauner, and P. M. Schulte, "Heat shock protein (Hsp70) induced by a mild heat shock slightly moderates plasma osmolarity increases upon salinity transfer in rainbow trout (Oncorhynchus mykiss)," Comparative Biochemistry and Physiology C, vol. 148, no. 4, pp. 437-444, 2008.

[34] M. Kozak, "An analysis of vertebrate mRNA sequences: intimations of translational control," Journal of Cell Biology, vol. 115, no. 4, pp. 887-903, 1991.

[35] N. Saha, L. M. Jyrwa, M. Das, and K. Biswas, "Influence of increased environmental water salinity on gluconeogenesis in the air-breathing walking catfish, Clarias batrachus," Fish Physiology and Biochemistry, vol. 37, no. 3, pp. 681-692, 2011.

[36] W. Luana, F. Li, B. Wang, X. Zhang, Y. Liu, and J. Xiang, "Molecular characteristics and expression analysis of calreticulin in Chinese shrimp Fenneropenaeus chinensis," Comparative Biochemistry and Physiology B, vol. 147, no. 3, pp. 482-491, 2007.

[37] H. Shimizu, H. Taniguchi, Y. Hippo, Y. Hayashizaki, H. Aburatani, and T. Ishikawa, "Characterization of the mouse Abcc12 gene and its transcript encoding an ATP-binding cassette transporter, an orthologue of human ABCC12," Gene, vol. 310, no. 1-2, pp. 17-28, 2003. 
[38] X. Wang, J. Dong, Y. Liu, and H. Gao, "A novel dehydrationresponsive element-binding protein from Caragana korshinskii is involved in the response to multiple abiotic stresses and enhances stress tolerance in transgenic tobacco," Plant Molecular Biology Reporter, vol. 28, no. 4, pp. 664-675, 2010.

[39] S. Bowen, C. Roberts, and A. E. Wheals, "Patterns of polymorphism and divergence in stress-related yeast proteins," Yeast, vol. 22, no. 8, pp. 659-668, 2005. 

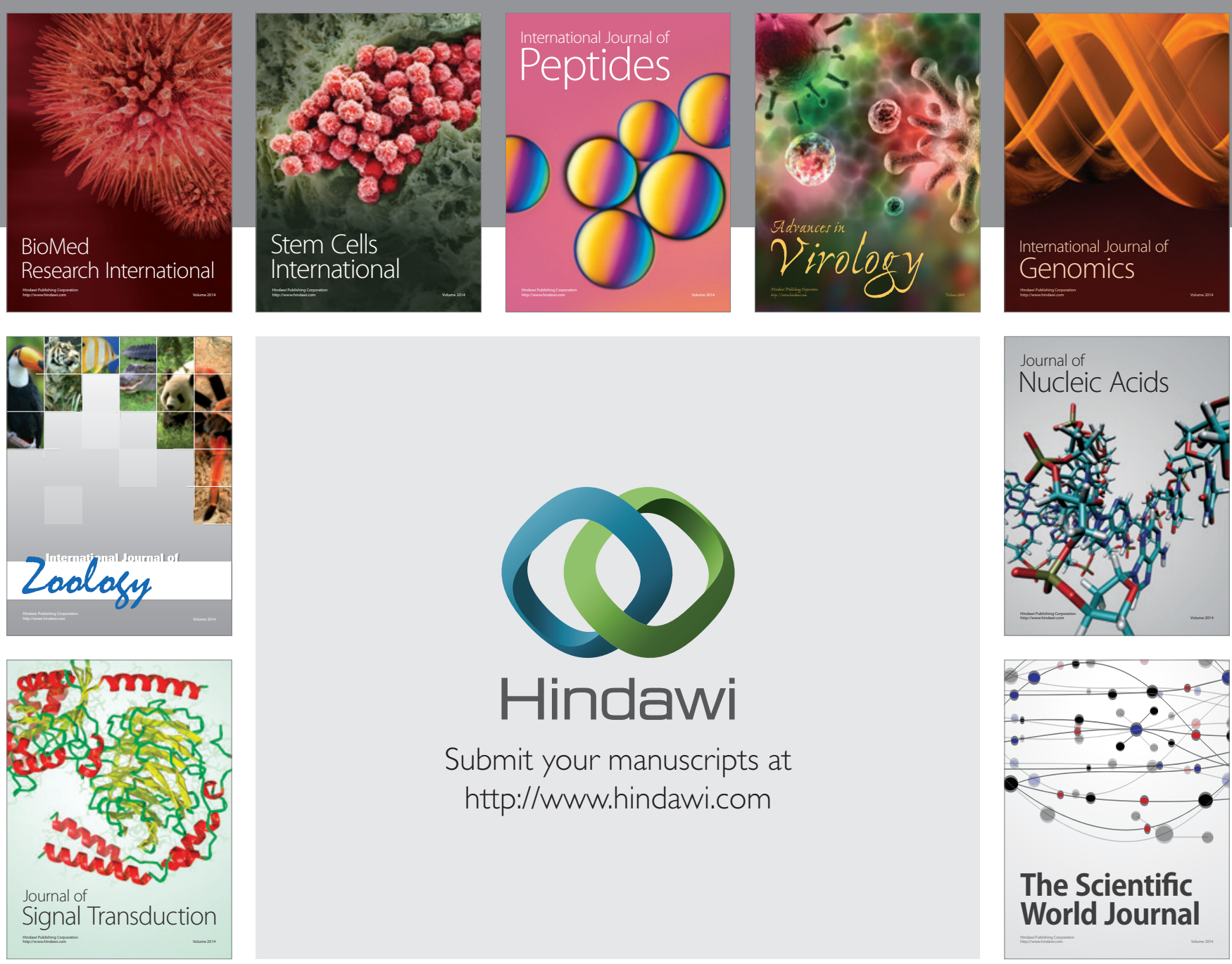

Submit your manuscripts at

http://www.hindawi.com
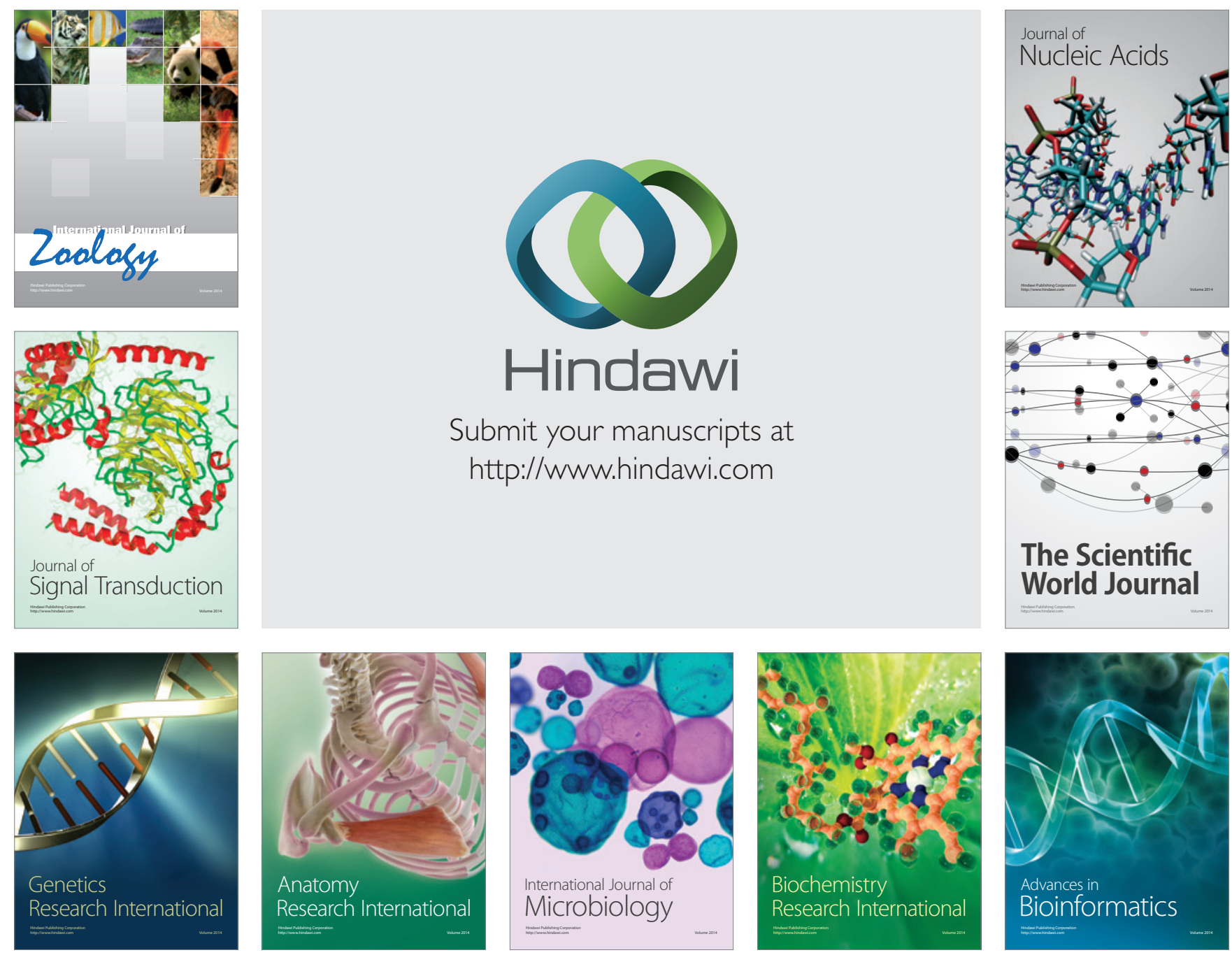

The Scientific World Journal
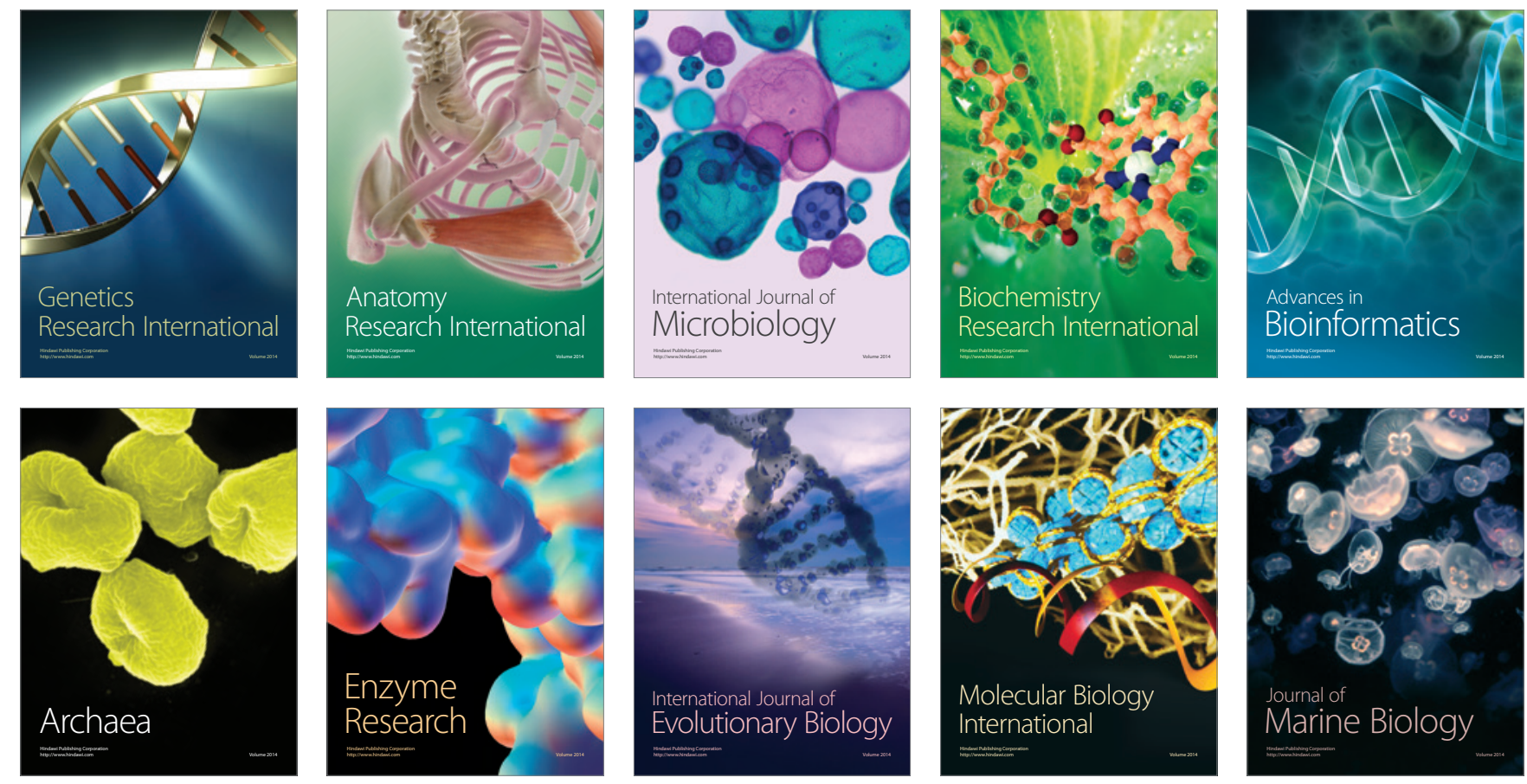